\title{
Economic differences among regional public service broadcasters in Spain according to their management model: An empirical analysis for period 2010-2013
}

\author{
Víctor Orive Serrano (D), María Pilar Latorre Martínez (D), Juan Pablo Artero MuñoziD \\ Universidad de Zaragoza (Spain) \\ orive@unizar.es,latorrep@unizar.es,jpartero@unizar.es
}

Received September, 2015

Accepted January, 2016

Versión en español

\section{Abstract}

Purpose: This piece of research quantifies and analyses empirically the given economic differences among public service television in Spain according to the adopted management model (classic or outsourced).

Design/methodology: In so doing, an average contrast of different economic variables studied in the literature is conducted (audience share, total assets, public subsidies, cost of personnel, suppliers spending and profit after taxes). In addition, these variables are related so as to calculate productivity obtained by each two groups of television operators. This analysis is conducted for period 2010-2013, featured by a crisis context in the Spanish economy.

Findings: Management model adopted by each regional broadcaster impacts on different economic variables as obtained share, total assets, public subsidies, cost of personnel, suppliers spending or profit after taxes. Moreover, those public corporations adopting an outsourced management model present better productivity values.

Research limitations/implications: Only one country has been analyzed for a 4 years period. 
Practical implications: Regional public service broadcasters with an outsourced model present less economic losses and require less public subsidies by their corresponding regional governments.

Social implications: Outsourcing part of the value chain can be useful so as to guarantee sustainability of regional public service television.

Originality/value: It has been proven empirically that the management model of a regional public service television impacts its economic results.

Keywords: Outsourcing, Management model, Productivity, Public service television, Regional television

Jel Codes: M1

\section{Introduction}

Public-service television networks face many economic, technological and social challenges that put their future and continuity in doubt and require an empirical analysis of how these public bodies are managed (Collins, Finn, Mcfadyen \& Hoskins, 2001; Bardoel \& D'Haenens, 2008; Iosifidis, 2010; Campos-Freire, 2013).

In Spain, national and regional public-service broadcasters have been criticized for different reasons, such as the lack of pluralism in the content they broadcast, their dependence on their government, the audience they achieve, their management efficiency, and the excessive budgetary deficits resulting from their production activity (Muñoz, 2011; Fernández Alonso \& Fernández Viso, 2012; Artero, Orive \& Latorre, 2015).

Regarding the latter, production activity, there are two different models for managing regional television networks: the traditional model and the outsourced model (Bustamante, 2009). These models show considerable differences that have been addressed from a theoretical perspective in the literature (Bustamante, 2009; López-Vidales, Azurmendi \& Órtiz, 2012; Sánchez \& Sarabia, 2012; Miguel de Bustos \& Casado, 2012). However, there are no empirical studies that analyze and compare these differences. 
Under these circumstances, the main purpose of this paper is to analyze and quantify the economic differences that characterize the regional public-service television bodies in Spain according to the management model they use (traditional or outsourced), for the 2010-2013 period, when Spain was in the midst of an economic crisis.

The article is organized as follows. Section 2 reviews the literature on the management of the production activity of the regional broadcasters. Then we describe the variables analyzed and the characteristics of the sample. Section 4 presents the research results. Finally, Section 5 contains the conclusions, limitations and future lines of research.

\section{Production management of regional broadcasters}

The approval of the Third Channel Act (46/1983-26 December) led the Autonomous Communities of Spain to create and develop their own regional public-service televisions. These publicly-owned bodies were conceived to provide relevant local content to their citizens and foster local audio-visual industries (Accenture, 2012).

Over the last three decades, 13 of the 17 regional governments have created regional public-service television networks. The time when they were created, together with political reasons, determined their production management models. Therefore, the regional public-service television networks developed during the first phase (1982-1989) chose the traditional management model, while the regional public bodies created in the second phase (1998-2006) adopted an outsourced management model.

The traditional model, followed by public broadcasters in the Basque Country, Catalonia, Galicia, Madrid and Andalusia, is based on the television channel assuming and carrying out all the activities that make up the audio-visual production value chain. This model mimics, at a regional level, the production structure of the Spanish state's public-service television (RTVE) and is linked with higher production costs and greater debt (Bustamante, 2009).

The outsourced model is based on subcontracting out to specialized suppliers one or more parts of the organization's value-chain activities (Fernández Alonso, 2002). The aim is to reduce production costs and avoid excessive indebtedness of public-service television (Sánchez \& Sarabia, 2012). This management model is used by the regional public television bodies of Asturias, Aragon, Castile-La Mancha, the Balearic Islands, Extremadura and Murcia. 
The economic literature published by the media has discussed the suitability of outsourcing as a management model for television companies. From a pro-outsourcing perspective, considerable cost savings (Alm \& Ferrell Lowe, 2003), greater flexibility and dynamism in the production structures (Cuesta, 2011) and a considerable growth and consolidation of the audio-visual industries in the autonomous communities where the television networks broadcast their contents are all noteworthy (Alm \& Ferrell Lowe, 2003).

However, there are also arguments against outsourcing. For example, McIvor, Humphreys and McAleer (1997) point out that it is difficult to properly measure and evaluate outsourcing because, when calculating cost savings, some qualitative aspects of subcontracting are omitted. Furthermore, outsourcing can decapitalize the channel since some of their potential production resources are dispensed with, possibly leading to an asymmetric relationship with suppliers (Cuesta, 2011).

Between 2010 and 2013, the 13 regional public-service networks received an annual public subsidy of over 3.033 billion euros. Moreover, they reached a share average of $7.92 \%$, the fourth most watched television group in Spain (Kantar Media, 2014). Regarding that aspect, it is important to specify that each channel broadcast its content to its own territory. Consequently, they do not compete for the same audiences among themselves.

However, they incurred financial losses of over 2 billion euros and cost each inhabitant 46 euros (Campos, 2012). Considering the high level of public debt and the deficit of the Spanish economy, these economic figures require the analysis of the production management of the regional broadcasters. To do this, we propose to investigate whether the management model adopted by the regional publicservice television can affect its economic performance.

\section{Analysis variables and sample characteristics}

The literature identifies three groups of variables that can be used to evaluate the economic performance of public television services: variables related to market share, financial variables and productivity variables (Picard, 2003). In this case, we have considered the following analytical variables: share of the main channel of the regional public service broadcaster within its own territory, total assets, public subsidies received, staff costs, materials costs and economic results. Tables 1 and 2 show the data on the 13 regional public-service television networks in Spain for the 2010-2013 period. 


\begin{tabular}{|c|c|c|c|c|c|c|c|}
\hline TV company & Year & $\begin{array}{c}\text { Share } \\
(\mathrm{x} 1)\end{array}$ & $\begin{array}{l}\text { Total Assets } \\
(\mathrm{x} 2)\end{array}$ & $\begin{array}{c}\text { Public } \\
\text { Subsidy }(\mathrm{x} 3) \\
\end{array}$ & Staff Costs (x4) & $\begin{array}{l}\text { Material Costs } \\
(\times 5)\end{array}$ & $\begin{array}{c}\text { Economic result } \\
(\mathrm{x} 6)\end{array}$ \\
\hline Andalucía & 2013 & 9.90 & $88,289,258$ & $111,462,964$ & $50,569,177$ & $37,358,449$ & $-15,429,424$ \\
\hline País Vasco & 2013 & 9.60 & $76,034,009$ & $97,877,401$ & $35,198,851$ & $37,127,915$ & $-6,818,033$ \\
\hline Cataluña & 2013 & 13.50 & $152,652,894$ & $330,273,366$ & $150,148,569$ & $79,456,133$ & $-16,037,533$ \\
\hline Galicia & 2013 & 10.90 & $87,659,897$ & $8,479,812$ & $45,587,962$ & $4,951,629$ & $-95,699,895$ \\
\hline Valencia & 2013 & 3.70 & n.d & n.d & n.d & n.d & n.d \\
\hline Madrid & 2013 & 3.80 & $45,857,872$ & $9,669,782$ & $69,377,363$ & $1,676,846$ & $-96,952,099$ \\
\hline Andalucía & 2012 & 10.10 & $49,042,773$ & $116,948,577$ & $52,154,083$ & $55,520,242$ & $-32,196,941$ \\
\hline País Vasco & 2012 & 9.90 & $92,380,376$ & $111,971,575$ & $34,122,525$ & $41,862,657$ & $-6,349,931$ \\
\hline Cataluña & 2012 & 14.30 & $191,585,852$ & $328,288,372$ & $120,175,327$ & $112,781,908$ & $-9,316,622$ \\
\hline Galicia & 2012 & 11.50 & $86,530,940$ & $7,740,768$ & $36,371,820$ & $4,390,538$ & $-88,369,778$ \\
\hline Valencia & 2012 & 5.00 & $83,626,532$ & $9,370,395$ & $73,993,446$ & $27,567,499$ & $-134,746,483$ \\
\hline Madrid & 2012 & 5.30 & $56,862,001$ & $15,763,222$ & $52,569,209$ & $30,826,923$ & $-115,140,041$ \\
\hline Andalucía & 2011 & 10.70 & $55,273,429$ & $155,572,670$ & $58,468,012$ & $73,115,657$ & $-22,752,243$ \\
\hline País Vasco & 2011 & 8.20 & $105,991,511$ & $131,607,331$ & $37,234,224$ & $59,367,545$ & $-5,640,112$ \\
\hline Cataluña & 2011 & 14.10 & $231,907,107$ & $368,536,088$ & $127,136,332$ & $91,506,987$ & $-8,770,358$ \\
\hline Galicia & 2011 & 12.30 & $94,615,128$ & $13,219,376$ & $40,505,882$ & $5,130,913$ & $-90,082,675$ \\
\hline Valencia & 2011 & 6.00 & $73,893,713$ & $17,360,690$ & $54,240,070$ & $59,912,200$ & $-136,845,392$ \\
\hline Madrid & 2011 & 6.40 & $77,782,645$ & $26,307,277$ & $50,841,180$ & $28,844,397$ & $-116,825,655$ \\
\hline Andalucía & 2010 & 12.70 & $79,535,847$ & $179,879,143$ & $58,554,921$ & $80,051,330$ & $-7,091,853$ \\
\hline País Vasco & 2010 & 9.40 & $112,348,191$ & $134,899,545$ & $38,611,040$ & $57,825,989$ & $-5,937,763$ \\
\hline Cataluña & 2010 & 14.80 & $260,913,203$ & $392,750,902$ & $132,221,702$ & $98,546,697$ & $-5,734,163$ \\
\hline Galicia & 2010 & 12.30 & $100,882,537$ & $17,550,584$ & $43,401,468$ & $6,022,830$ & $-97,065,941$ \\
\hline Valencia & 2010 & 8.40 & $119,163,067$ & $25,112,767$ & $58,975,585$ & $71,765,791$ & $-164,649,475$ \\
\hline Madrid & 2010 & 8.00 & $89,133,252$ & $35,939,545$ & $51,563,797$ & $25,117,634$ & $-101,997,671$ \\
\hline
\end{tabular}

Table 1. Regional public services with traditional management model (financial statements)

\begin{tabular}{|c|c|c|c|c|c|c|c|}
\hline TV company & Year & Share $(x 1)$ & Total Assets (x2) & $\begin{array}{c}\text { Public Subsidy } \\
(\mathrm{x} 3)\end{array}$ & $\begin{array}{c}\text { Staff } \\
\text { Costs }(x 4)\end{array}$ & $\begin{array}{c}\text { Material } \\
\text { Costs (x5) }\end{array}$ & $\begin{array}{l}\text { Economic } \\
\text { result }(x 6)\end{array}$ \\
\hline Aragón & 2013 & 11.50 & $30,067,140$ & $39,129,386$ & $2,858,184$ & $21,141,237$ & 361,186 \\
\hline Asturias & 2013 & 5.40 & $14,991,766$ & 901,990 & $4,235,456$ & $11,031,426$ & $-17,859,503$ \\
\hline Murcia & 2013 & n.d & n.d & n.d & n.d & n.d & n.d \\
\hline Castilla la Mancha & 2013 & 4.40 & $18,425,817$ & $31,262,711$ & $12,451,144$ & $10,653,960$ & $-130,443,000$ \\
\hline Extremadura & 2013 & 4.50 & $14,971,110$ & 703,729 & $7,590,072$ & $9,218,978$ & $-22,391,486$ \\
\hline Baleares & 2013 & 5.90 & $28,572,056$ & $24,286,645$ & $2,459,755$ & $18,060,646$ & $-6,504,289$ \\
\hline Canarias & 2013 & 7.20 & n.d & n.d & n.d & n.d & n.d \\
\hline Aragón & 2012 & 11.30 & $29,157,898$ & $2,788,832$ & $2,734,472$ & $22,492,618$ & $-38,258,149$ \\
\hline Asturias & 2012 & 6.90 & $20,141,307$ & 946,722 & $4,901,810$ & $20,730,011$ & $-30,633,611$ \\
\hline Murcia & 2012 & 2.50 & $6,448,934$ & 488,247 & 492,461 & $20,284,501$ & $-27,304,122$ \\
\hline Castilla la Mancha & 2012 & 5.10 & $23,296,785$ & $30,814,463$ & $13,164,214$ & $13,120,024$ & n.d \\
\hline Extremadura & 2012 & 1.50 & $18,188,818$ & 733,663 & $6,837,072$ & $12,158,163$ & $-24,628,049$ \\
\hline Baleares & 2012 & 6.30 & $40,530,477$ & $34,214,813$ & $2,577,324$ & $22,729,479$ & $-10,623,843$ \\
\hline Canarias & 2012 & 7.60 & n.d & n.d & n.d & n.d & n.d \\
\hline Aragón & 2011 & 10.70 & $35,081,491$ & $3,135,158$ & $3,172,959$ & $32,550,729$ & $-49,926,021$ \\
\hline Asturias & 2011 & 7.70 & $32,613,764$ & $2,813,485$ & $4,483,149$ & $24,923,490$ & $-28,525,235$ \\
\hline Murcia & 2011 & 4.00 & $26,459,997$ & $1,474,147$ & 563,414 & $24,995,901$ & $-28,583,275$ \\
\hline Castilla la Mancha & 2011 & 6.40 & $30,347,672$ & $49,937,654$ & $13,755,287$ & $22,163,413$ & n.d \\
\hline Extremadura & 2011 & 1.70 & $11,373,831$ & $1,774,293$ & $7,219,311$ & $15,664,906$ & $-24,641,414$ \\
\hline Baleares & 2011 & 5.20 & $57,682,048$ & $31,820,846$ & $2,577,324$ & $31,117,292$ & $-9,532,359$ \\
\hline Canarias & 2011 & 7.90 & $20,450,409$ & $6,549,246$ & $3,292,542$ & $37,379,859$ & $-41,337,570$ \\
\hline Aragón & 2010 & 9.40 & $25,466,274$ & $3,888,158$ & $3,068,570$ & $34,878,180$ & $-55,095,087$ \\
\hline Asturias & 2010 & 7.60 & $19,474,788$ & $3,252,084$ & $4,336,784$ & $24,824,396$ & $-28,259,432$ \\
\hline Murcia & 2010 & 4.60 & $19,808,132$ & $1,868,005$ & 520,176 & $37,923,350$ & $-42,082,548$ \\
\hline Castilla la Mancha & 2010 & 7.50 & $24,077,150$ & $54,034,835$ & $13,665,929$ & $24,039,573$ & n.d \\
\hline Extremadura & 2010 & 5.00 & $12,403,930$ & $4,511,259$ & $4,457,372$ & $18,548,472$ & $-21,195,949$ \\
\hline Baleares & 2010 & 5.50 & $50,521,137$ & $44,334,853$ & $2,767,890$ & $29,839,788$ & $-4,146,758$ \\
\hline Canarias & 2010 & 10.00 & $32,295,891$ & $11,059,493$ & $3,417,525$ & $41,127,229$ & $-43,173,646$ \\
\hline
\end{tabular}

Tabla 2. Regional public services with outsourced management model (financial statements) 


\section{Results}

To analyze the economic differences of the regional televisions according to their management models, we first compared differences between the means of the two groups of broadcasters. Then, the productivity levels of the broadcasters were analyzed based on their management models. SPSS 20.0 statistics software was used to perform the analysis.

\subsection{Comparison of means differences}

As shown, public broadcasters using a traditional management model show far higher mean values than public bodies with an outsourced management model in most of the economic variables analyzed (see Table 3).

Therefore, overall, regional televisions with a traditional management model achieve a greater share and a greater volume of assets. Furthermore, they also receive a greater public subsidy from their regional government; likewise, these broadcasters spend more on staff and materials. However, corporations adopting a traditional model have twice the losses of those television networks that outsource their activities.

All these differences obtained in the mean values of the variables are statistically significant. Therefore, with a confidence level of $95 \%$, the t-Student test for independent samples reveals that the management model adopted by regional public-service televisions affects the share obtained, the total amount of assets, the public subsidy received, staff costs, materials costs and the economic result.

\begin{tabular}{|l|r|r|r|r|}
\hline \multicolumn{1}{|c|}{ Variable compared } & Traditional model & Outsourced model & T-value & p-value \\
\hline (x1) Share & 9.62 & 6.19 & 45.99 & $0.000^{*}$ \\
\hline (x2) Total Assets & $100,498,418.00$ & $22,958,879.40$ & -6.392 & $0.000^{*}$ \\
\hline (x3) Public Subsidy & $110,274,256.00$ & $13,811,597.00$ & -3.748 & $0.001^{*}$ \\
\hline (x4) Staff Costs & $61,334,272.71$ & $45,577,149.85$ & -7.736 & $0.000^{*}$ \\
\hline (x5) Material Costs & $45,447,029.54$ & $20,771,343.61$ & -3.448 & $0.000^{*}$ \\
\hline (x6) Economic Result & $-57,518,753.40$ & $-24,456,577.10$ & 32.379 & $0.011^{*}$ \\
\hline * Significat for $\mathrm{p}<0,05$ & \multicolumn{4}{|r}{}
\end{tabular}

Table 3. Analysis of the differences between means according to the management model (2010-2013) 


\subsection{Productivity analysis}

Table 4 shows the productivity values obtained by regional channels based on the management model used. These values use different variables such as the volume of assets, capital received, labor or materials used (Picard, 2003) to indicate how effective a company is at obtaining products and services (Brinkerhoff \& Dressler, 1990).

As shown, each share point obtained by the public networks with traditional management models requires a volume of assets 3 times higher than the networks with an outsourcing model.

Furthermore, over the 2010-2013 period, regional governments had to spend five times more for each share point on the group of regional broadcasters with traditional management models than on the group of broadcasters with an outsourced management model.

Regarding the relationship between staff costs and share, public bodies using outsourcing need 1 million euros more in staff costs per share point than traditional public networks. However, the regional public-service broadcasters with a traditional management model spent 1.5 million euros more in material costs for each share point than the regional broadcasters with an outsourced management model.

\begin{tabular}{|l|r|r|}
\cline { 2 - 3 } \multicolumn{1}{c|}{} & Traditional model & Outsourced model \\
\hline (x2) Total assets / Share (x3) & $10,446,821$ & $3,709,027.37$ \\
\hline (x3) Public Subsidy / (x1) Share & $11,463,020.37$ & $2,231,275.77$ \\
\hline (x4) Staff Costs / (x1) Share & $6,375,704.02$ & $7,363,029.05$ \\
\hline (x5) Material Costs / (x1) Share & $4,724,223.445$ & $3,355,629.02$ \\
\hline
\end{tabular}

Tabla 4. Productivity according to the management model (2010-2013)

\section{Conclusions}

Public television services are currently affected by many threats of several kinds. Among these threats is the economic crisis that questions whether these public entities are sustainable given the income and expenses resulting from their activities and the economic deficits they generate.

In this context, this paper analyzes the influence of the management model adopted by public television networks to manage their production activities, using several economic variables.

The research results reveal that public television entities that use a management model based on outsourcing part of their production activities have lower economic losses and require less public 
subsidies. In addition, this management strategy achieves much better productivity levels than those obtained by the management model that opposes outsourcing. In this regard, public corporations that opt for outsourcing need lower levels of assets and financial subsidies for each share point gained.

However, outsourcing as a management strategy also has certain disadvantages which should be noted. For example, public-service television networks following an outsourcing strategy obtained much smaller absolute share, and require a greater outlay in staff expenses to obtain a share point. Furthermore, as literature highlights, outsourcing might imply important negative effects that have to be taken into account when considering that management model.

Finally, this work presents some limitations that must be taken into account. In this regard, it is noted that the investigation analyzed a single country over a period of 4 years and has just included regional public service broadcasters.

Therefore, future lines of research should be directed towards analyzing the economic management of public television services in other European countries over longer periods. In addition, it can be highly relevant to expand that analysis to national and local television channels.

\section{References}

Accenture (2012). Informe sobre el papel de la televisión pública autonómica en España. Madrid.

Alm, A., \& Ferrell Lowe, G. (2003). Outsourcing core competencies?. En G. Ferrel Lowe \& T. Hujanen (eds.), Broadcasting and convergence: New articulations of the public service remit. Goterborg: Nordicom.

Artero, J.P., Orive, V., \& Latorre, P. (2015). Efficiency and benchmarks of regional public service broadcasters in Spain. Communication \& Society, 28(3), 13-30. http://dx.doi.org/10.15581/003.28.3.13-28

Bardoel, J., \& D’Haenens, L. (2008). Reinventing public service broadcasting in Europe: Prospect, promises and problems. Media, Culture and Society, 30(3), 337-355. http://dx.doi.org/10.1177/0163443708088791

Brinkerhoff, R., \& Dressler, D. (1990). Productivity Measurement. A Guide for Managers and Evaluators. London: Sage.

Bustamante, E. (2009). El audiovisual digital: La televisión regional ante el reto de futuro. En E. Bustamante (Ed.), El audiovisual digital. Politicas y estrategias desde las Comunidades Autónomas. Tenerife: IDECO. 
Campos, F. (2012). Modelo de financiación de las televisiones autonómicas. En J.C. Miguel \& M.A Casado (coords.), Televisiones autonómicas. Evolución y crisis del modelo público de proximidad. Gedisa: Barcelona.

Campos-Freire, F. (2013). El futuro de la TV europea es híbrido, convergente y cada vez menos público. Revista Latina de Comunicación Social, 68, 89-118. http://dx.doi.org/10.4185/RLCS-2013-970

Collins, R., Finn, R., Mcfadyen, S., \& Hoskins, C. (2001). Public service broadcasting beyond 2000: Is there a future for public service broadcasting?. Canadian Journal of Communication, 26(1), 3-15.

Cuesta, U. (2011). Las televisiones autonómicas en el nuevo panorama audiovisual. Documento para el debate. Madrid: Fundación Ciudadanía y Valores. available online at: http://www.funciva.org/uploads/ficheros documentos/1304077495 ponencia ii.pdf

Fernández Alonso, I. (2002). La externalización de la producción de los operadores públicos de televisión de ámbito autonómico en España. Los casos canario y extremeño. Revista Latina de Comunicación Social, 46. Available online at: http://www.ull.es/publicaciones/latina/2002/latina46enero/4604isabel.htm

Fernández Alonso, I., \& Fernández Viso, A. (2012). Internal pluralism in the governance of public service broadcasters in Spain and the role of social groups and professionals. The case of RTVE. Communication \& Society, 25(2), 203-230.

Kantar Media (2014). Informe anual de la audiencia de televisión. Madrid

Iosifidis, P. (2010). Review: M.Debrett, Reinventing public service televisión for digital future. International Journal of Digital Television, 2(1), 1-3. http://dx.doi.org/10.1386/jdtv.2.1.123_5

López-Vidales, N., Azurmendi, A., \& Órtiz, M.Á. (2012). Hacia un nuevo marco regulatorio y de gestión de la televisión pública en España: El bienio del cambio. Revista de Comunicación de la SEECI, 27, 39-59. http://dx.doi.org/10.15198/seeci.2012.27.39-59

Mcivor, R., Humphreys, P.K, \& Mcaleer, W. (1997). A strategic model for the formulation of and effective make or buy decision. Management Decision, 35(2), 169-178. http://dx.doi.org/10.1108/00251749710160331

Miguel De Bustos, J.C., \& Casado, M.A. (2012). Televisiones autonómicas: Evolución y crisis del modelo público de proximidad. Barcelona: Gedisa.

Muñoz, M. (2011). Key issues to understand the controversy surrounding the economic support of Spanish public television. Communication \& Society, 24(1), 119-140.

Picard, R. (2003). Assessment of Public Service Broadcasting: Economic and Managerial Performance Criteria. Javnost - The Public, 10(3), 29-44. http://dx.doi.org/10.1080/13183222.2003.11008833 
Sánchez, J., \& Sarabia, I. (2012). Las estructuras de Gobierno de los nuevos modelos de televisión autonómica pública en España. Gestión y Análisis de las Políticas Públicas, 8, 35-61.

\section{Versión en español}

Título: Diferencias económicas de las televisiones autonómicas en España según el modelo de gestión: Un análisis empírico para el periodo 2010-2013

\section{Resumen}

Objeto: Este trabajo cuantifica y analiza empíricamente las diferencias económicas existentes entre las televisiones públicas en España según el modelo de gestión adoptado por el ente público (modelo clásico o modelo externalizado).

Diseño/metodología/enfoque: Para ello, se realiza un contraste de medias de diferentes variables económicas recogidas en la literatura (share, volumen total de activos, subvención pública recibida, gastos de personal, gastos en materiales y resultado económico) entre los dos grupos de entes públicos regionales según el modelo de gestión. Además, se relacionan dichas variables para calcular la productividad obtenida para cada grupo de televisiones. Todo ello para el periodo 2010-2013, caracterizado por el contexto de crisis de la economía española.

Aportaciones y resultados: El modelo de gestión adoptado por la cadena de televisión autonómica influye en diferentes variables económicas como el share obtenido, la cifra total de activos, la subvención pública recibida, los gastos en personal, los gastos desembolsados en materiales o el resultado económico. Además, los entes públicos que utilizan un modelo de gestión basado en la externalización de su actividad presentan mejores valores de productividad.

Limitaciones: Se ha analizado un único país para un periodo temporal de 4 años.

Implicaciones prácticas: Las televisiones autonómicas con un modelo de externalización presentan menores pérdidas económicas y requieren de una menor asignación pública de los Gobiernos correspondientes.

Implicaciones sociales: La externalización de parte de la actividad de la cadena de televisión autonómica puede ser útil para garantizar la sostenibilidad del servicio público regional de televisión. 
Originalidad / Valor añadido: Se ha comprobado empíricamente que el modelo de gestión que adopta una televisión autonómica pública influye en el resultado económico.

Palabras clave: Outsourcing, Management model, Productivity, Public service television, Regional television

\section{Códigos JEL: M1}

\section{Introducción}

Los servicios públicos de televisión se enfrentan a numerosos retos económicos, tecnológicos y sociales que cuestionan su futuro y continuidad y que obligan a analizar desde una perspectiva empírica la gestión de los entes públicos (Collins, Finn, Mcfadyen \& Hoskins, 2001; Bardoel \& D’Haenens, 2008; Iosidifis, 2010; Campos-Freire, 2013).

En el contexto español, los servicios públicos nacionales y autonómicos de televisión se han visto cuestionados por diferentes razones como por ejemplo la falta de pluralismo en los contenidos emitidos, la dependencia hacia el Gobierno correspondiente, la audiencia obtenida, la eficiencia en la gestión, o el excesivo déficit económico derivado del desempeño su actividad productiva (Muñoz, 2011; Fernández Alonso \& Fernández Viso, 2012; Artero, Orive \& Latorre, 2015).

Con respecto a este último aspecto, el desempeño de la actividad productiva, se distinguen dos modelos gerenciales diferentes para gestionar las televisiones autonómicas: el modelo clásico y el modelo externalizado (Bustamante, 2009). Dichos modelos presentan considerables diferencias que la literatura ha abordado desde una perspectiva teórica (Bustamante, 2009; López-Vidales, Azurmendi \& Órtiz, 2012; Sánchez \& Sarabia, 2012; Miguel de Bustos \& Casado, 2012). Sin embargo, no existen trabajos empíricos que analicen y contrasten dichas diferencias.

Ante esta situación, este trabajo tiene como objetivo principal analizar y cuantificar las diferencias económicas que caracterizan a los servicios públicos autonómicos de televisión en España según el modelo de gestión utilizado (clásico o externalizado). Todo ello para el periodo 2010-2013 caracterizado por el contexto de crisis económica en España. 
El artículo se estructura de la siguiente manera. En la sección 2 se realiza una revisión de la literatura sobre la gestión de la actividad productiva de las televisiones autonómicas. A continuación, se describen las variables de análisis y las características de la muestra. En la sección 4 se presentan los resultados de la investigación. Por último, la sección 5 recoge las conclusiones, las limitaciones y las futuras líneas de investigación.

\section{Gestión productiva de las televisiones autonómicas}

La aprobación de la Ley del Tercer Canal (46/1983-26 de Diciembre) dio origen al desarrollo y creación por parte de las Comunidades Autónomas de diferentes servicios públicos regionales de televisión en España. Estos entes, de titularidad pública, fueron ideados con el objetivo de ofrecer contenidos de proximidad a sus ciudadanos y de dinamizar la industria audiovisual local (Accenture, 2012).

A lo largo de las últimas tres décadas 13 de los 17 Gobiernos autonómicos han creado un servicio público regional de televisión. El periodo temporal en el que se creó el ente, unido a razones políticas, ha determinado el modelo de gestión de la actividad productiva utilizado por los gestores. Así, los servicios públicos autonómicos de televisión desarrollados en una primera fase (1982-1989) optaron por un modelo de gestión clásico mientras que los entes públicos autonómicos creados en una segunda fase (1998-2006) adoptaron un modelo de gestión externalizado.

El modelo clásico, seguido por las televisiones autonómicas de País Vasco, Cataluña, Galicia, Madrid y Andalucía, se basa en que la cadena de televisión asume y realiza la totalidad de las actividades que forman la cadena de valor del producto audiovisual. Este modelo imita a escala autonómica la estructura productiva del servicio público nacional de televisión (RTVE) y está asociado a unos mayores costes productivos y a una mayor deuda (Bustamante, 2009).

El modelo externalizado se basa en subcontratar a suministradores especializados una o varias partes de la actividades de la cadena de valor de la organización (Fernández Alonso, 2002). Todo ello con el objetivo de reducir costes productivos y evitar un excesivo endeudamiento del servicio público de televisión (Sánchez \& Sarabia, 2012). Este modelo de gestión es utilizado por los entes públicos autonómicos de televisión de Asturias, Aragón, Castilla la Macha, Baleares, Canarias, Extremadura y Murcia

La literatura de economía de los medios ha debatido acerca de la idoneidad de la externalización como modelo de gestión de las empresas de televisión. Desde una perspectiva favorable a la externalización, 
se destaca el considerable ahorro en costes (Alm \& Ferrell, 2003), una mayor flexibilidad y dinamismo en las estructuras productivas para los entes (Cuesta, 2011) y un considerable impulso y consolidación a las industrias audiovisuales regionales donde las cadenas de televisión emiten sus contenidos (Alm \& Ferrell Lowe, 2003).

Sin embargo, también existen argumentos contrarios a la externalización. Así, McIvor, Humphreys y McAleer (1997) señalan que es difícil medir y evaluar correctamente la externalización ya que en el cálculo de ahorro en costes se obvian algunos aspectos cualitativos derivados de la subcontratación. Además, la externalización puede generar descapitalización interna de la cadena, ya que prescinde de algunos de sus potenciales recursos productivos, y puede crear una posible relación asimétrica con los proveedores (Cuesta, 2011).

Durante el periodo 2010-2013 los 13 servicios públicos autonómicos de televisión y recibieron una asignación pública anual superior a los 3,033 millones de euros. Además, promediaron en su conjunto un $7.92 \%$ de share situándose como cuarto grupo televisivo más visto en España (Kantar Media, 2014). En este sentido, es importante especificar que cada ente público autonómico de televisión emite sus contenidos en su Comunidad Autónoma de origen correspondiente, y que por lo tanto no compiten entre ellos por la audiencia en un mismo territorio.

Sin embargo, incurrieron en unas pérdidas económicas superiores a los 2,000 millones de euros y tuvieron un coste por habitante de 46 euros (Campos, 2012)

Estas cifras económicas obtenidas por los entes, y teniendo en cuenta el escenario de elevada deuda y déficit público que presenta la economía española, obligan a analizar la gestión productiva de las cadenas de televisión autonómicas. Para ello, se propone investigar si el modelo de gestión adoptado por el servicio público autonómico de televisión puede tener influencia en el rendimiento económico.

\section{Variables de análisis y características de la muestra}

La literatura señala tres grupos de variables para evaluar el rendimiento económico de los servicios públicos de televisión: variables relativas a la cuota de mercado, variables financieras y variables de productividad (Picard, 2003). En este sentido, se han considerado las siguientes variables de análisis: share del canal principal de televisión del ente público autonómico que obtiene mayor cuota de pantalla en su comunidad autónoma de origen, volumen total de activos, subvención pública recibida, gastos de 
personal, gastos en materiales y resultado económico. En la Tablas 1 y 2 se presentan los datos relativos a los 13 servicios públicos autonómicos de televisión en España durante el periodo 2010-2013.

\begin{tabular}{|c|c|c|c|c|c|c|c|}
\hline $\begin{array}{l}\text { Empresa } \\
\text { televisión }\end{array}$ & Año & $\begin{array}{c}\text { Share } \\
(\mathrm{x} 1)\end{array}$ & $\begin{array}{l}\text { Total Activo } \\
(\mathrm{x} 2)\end{array}$ & $\begin{array}{l}\text { Subvención } \\
\text { pública }(x 3)\end{array}$ & $\begin{array}{c}\text { Gastos personal } \\
(\mathrm{x} 4)\end{array}$ & $\begin{array}{c}\text { Gastos } \\
\text { materiales (x5) }\end{array}$ & $\begin{array}{c}\text { Resultado } \\
\text { económico }(\mathrm{x} 6)\end{array}$ \\
\hline Andalucía & 2013 & 9.90 & $88,289,258$ & $111,462,964$ & $50,569,177$ & $37,358,449$ & $-15,429,424$ \\
\hline País Vasco & 2013 & 9.60 & $76,034,009$ & $97,877,401$ & $35,198,851$ & $37,127,915$ & $-6,818,033$ \\
\hline Cataluña & 2013 & 13.50 & $152,652,894$ & $330,273,366$ & $150,148,569$ & $79,456,133$ & $-16,037,533$ \\
\hline Galicia & 2013 & 10.90 & $87,659,897$ & $8,479,812$ & $45,587,962$ & $4,951,629$ & $-95,699,895$ \\
\hline Valencia & 2013 & 3.70 & n.d & n.d & n.d & n.d & n.d \\
\hline Madrid & 2013 & 3.80 & $45,857,872$ & $9,669,782$ & $69,377,363$ & $1,676,846$ & $-96,952,099$ \\
\hline Andalucía & 2012 & 10.10 & $49,042,773$ & $116,948,577$ & $52,154,083$ & $55,520,242$ & $-32,196,941$ \\
\hline País Vasco & 2012 & 9.90 & $92,380,376$ & $111,971,575$ & $34,122,525$ & $41,862,657$ & $-6,349,931$ \\
\hline Cataluña & 2012 & 14.30 & $191,585,852$ & $328,288,372$ & $120,175,327$ & $112,781,908$ & $-9,316,622$ \\
\hline Galicia & 2012 & 11.50 & $86,530,940$ & $7,740,768$ & $36,371,820$ & $4,390,538$ & $-88,369,778$ \\
\hline Valencia & 2012 & 5.00 & $83,626,532$ & $9,370,395$ & $73,993,446$ & $27,567,499$ & $-134,746,483$ \\
\hline Madrid & 2012 & 5.30 & $56,862,001$ & $15,763,222$ & $52,569,209$ & $30,826,923$ & $-115,140,041$ \\
\hline Andalucía & 2011 & 10.70 & $55,273,429$ & $155,572,670$ & $58,468,012$ & $73,115,657$ & $-22,752,243$ \\
\hline País Vasco & 2011 & 8.20 & $105,991,511$ & $131,607,331$ & $37,234,224$ & $59,367,545$ & $-5,640,112$ \\
\hline Cataluña & 2011 & 14.10 & $231,907,107$ & $368,536,088$ & $127,136,332$ & $91,506,987$ & $-8,770,358$ \\
\hline Galicia & 2011 & 12.30 & $94,615,128$ & $13,219,376$ & $40,505,882$ & $5,130,913$ & $-90,082,675$ \\
\hline Valencia & 2011 & 6.00 & $73,893,713$ & $17,360,690$ & $54,240,070$ & $59,912,200$ & $-136,845,392$ \\
\hline Madrid & 2011 & 6.40 & $77,782,645$ & $26,307,277$ & $50,841,180$ & $28,844,397$ & $-116,825,655$ \\
\hline Andalucía & 2010 & 12.70 & $79,535,847$ & $179,879,143$ & $58,554,921$ & $80,051,330$ & $-7,091,853$ \\
\hline País Vasco & 2010 & 9.40 & $112,348,191$ & $134,899,545$ & $38,611,040$ & $57,825,989$ & $-5,937,763$ \\
\hline Cataluña & 2010 & 14.80 & $260,913,203$ & $392,750,902$ & $132,221,702$ & $98,546,697$ & $-5,734,163$ \\
\hline Galicia & 2010 & 12.30 & $100,882,537$ & $17,550,584$ & $43,401,468$ & $6,022,830$ & $-97,065,941$ \\
\hline Valencia & 2010 & 8.40 & $119,163,067$ & $25,112,767$ & $58,975,585$ & $71,765,791$ & $-164,649,475$ \\
\hline Madrid & 2010 & 8.00 & $89,133,252$ & $35,939,545$ & $51,563,797$ & $25,117,634$ & $-101,997,671$ \\
\hline
\end{tabular}

Tabla 1. Servicios públicos autonómicos con un modelo de gestión clásico (Cuentas Anuales)

\begin{tabular}{|c|c|c|c|c|c|c|c|}
\hline Empresa televisión & Año & Share (x1) & $\begin{array}{c}\text { Total Activo } \\
(\mathrm{x} 2)\end{array}$ & $\begin{array}{l}\text { Subvención } \\
\text { pública }(x 3)\end{array}$ & $\begin{array}{c}\text { Gastos } \\
\text { personal }(x 4)\end{array}$ & $\begin{array}{c}\text { Gastos } \\
\text { materiales (x5) }\end{array}$ & $\begin{array}{c}\text { Resultado } \\
\text { económico }(\mathrm{x} 6)\end{array}$ \\
\hline Aragón & 2013 & 11.50 & $30,067,140$ & $39,129,386$ & $2,858,184$ & $21,141,237$ & 361,186 \\
\hline Asturias & 2013 & 5.40 & $14,991,766$ & 901,990 & $4,235,456$ & $11,031,426$ & $-17,859,503$ \\
\hline Murcia & 2013 & n.d & n.d & n.d & n.d & n.d & n.d \\
\hline Castilla la Mancha & 2013 & 4.40 & $18,425,817$ & $31,262,711$ & $12,451,144$ & $10,653,960$ & $-130,443,000$ \\
\hline Extremadura & 2013 & 4.50 & $14,971,110$ & 703,729 & $7,590,072$ & $9,218,978$ & $-22,391,486$ \\
\hline Baleares & 2013 & 5.90 & $28,572,056$ & $24,286,645$ & $2,459,755$ & $18,060,646$ & $-6,504,289$ \\
\hline Canarias & 2013 & 7.20 & n.d & n.d & n.d & n.d & n.d \\
\hline Aragón & 2012 & 11.30 & $29,157,898$ & $2,788,832$ & $2,734,472$ & $22,492,618$ & $-38,258,149$ \\
\hline Asturias & 2012 & 6.90 & $20,141,307$ & 946,722 & $4,901,810$ & $20,730,011$ & $-30,633,611$ \\
\hline Murcia & 2012 & 2.50 & $6,448,934$ & $488,25 €$ & 492,461 & $20,284,501$ & $-27,304,122$ \\
\hline Castilla la Mancha & 2012 & 5.10 & $23,296,785$ & $30,814,463$ & $13,164,214$ & $13,120,024$ & n.d \\
\hline Extremadura & 2012 & 1.50 & $18,188,818$ & 733,663 & $6,837,072$ & $12,158,163$ & $-24,628,049$ \\
\hline Baleares & 2012 & 6.30 & $40,530,477$ & $34,214,813$ & $2,577,324$ & $22,729,479$ & $-10,623,843$ \\
\hline Canarias & 2012 & 7.60 & n.d & n.d & n.d & n.d & n.d \\
\hline Aragón & 2011 & 10.70 & $35,081,491$ & $3,135,158$ & $3,172,959$ & $32,550,729$ & $-49,926,021$ \\
\hline Asturias & 2011 & 7.70 & $32,613,764$ & $2,813,485$ & $4,483,149$ & $24,923,490$ & $-28,525,235$ \\
\hline Murcia & 2011 & 4.00 & $26,459,997$ & $1,474,147$ & 563,414 & $24,995,901$ & $-28,583,275$ \\
\hline Castilla la Mancha & 2011 & 6.40 & $30,347,672$ & $49,937,654$ & $13,755,287$ & $22,163,413$ & n.d \\
\hline Extremadura & 2011 & 1.70 & $11,373,831$ & $1,774,293$ & $7,219,311$ & $15,664,906$ & $-24,641,414$ \\
\hline Baleares & 2011 & 5.20 & $57,682,048$ & $31,820,846$ & $2,577,324$ & $31,117,292$ & $-9,532,359$ \\
\hline Canarias & 2011 & 7.90 & $20,450,409$ & $6,549,246$ & $3,292,542$ & $37,379,859$ & $-41,337,570$ \\
\hline Aragón & 2010 & 9.40 & $25,466,274$ & $3,888,158$ & $3,068,570$ & $34,878,180$ & $-55,095,087$ \\
\hline Asturias & 2010 & 7.60 & $19,474,788$ & $3,252,084$ & $4,336,784$ & $24,824,396$ & $-28,259,432$ \\
\hline Murcia & 2010 & 4.60 & $19,808,132$ & $1,868,005$ & 520,176 & $37,923,350$ & $-42,082,548$ \\
\hline Castilla la Mancha & 2010 & 7.50 & $24,077,150$ & $54,034,835$ & $13,665,929$ & $24,039,573$ & n.d \\
\hline Extremadura & 2010 & 5.00 & $12,403,930$ & $4,511,259$ & $4,457,372$ & $18,548,472$ & $-21,195,949$ \\
\hline Baleares & 2010 & 5.50 & $50,521,137$ & $44,334,853$ & $2,767,890$ & $29,839,788$ & $-4,146,758$ \\
\hline Canarias & 2010 & 10.00 & $32,295,891$ & $11,059,493$ & $3,417,525$ & $41,127,229$ & $-43,173,646$ \\
\hline
\end{tabular}

Tabla 2. Servicios públicos autonómicos con un modelo de gestión externalizado (Cuentas Anuales) 


\section{Resultados}

Para analizar las diferencias económicas de las televisiones autonómicas según el modelo de gestión adoptado, se realiza en primer lugar un contraste de diferencias de medias entre los dos grupos de cadenas. A continuación se analiza la productividad de los entes televisivos según el modelo de gestión. Para llevar a cabo dichos análisis se ha utilizado el programa estadístico SPSS 20.0.

\subsection{Contraste de diferencia de medias}

Como puede observarse, los entes públicos que siguen un modelo de gestión clásico presentan valores medios muy superiores a los que tienen los entes públicos con un modelo de gestión externalizado en la mayoría de variables económicas analizadas (ver Tabla 3).

Así, en su conjunto, las televisiones autonómicas con un modelo de gestión clásico consiguen un mayor share y presentan un mayor volumen de activos. Además, reciben una mayor subvención pública por parte de la administración pública regional correspondiente. Asimismo, estas cadenas de televisión tienen unos mayores gastos en personal y en materiales. Sin embargo, el resultado económico de las corporaciones con un modelo clásico arroja unas pérdidas que duplican a las obtenidas por el grupo de cadenas de televisión que externaliza su actividad.

Todas estas diferencias obtenidas en los valores medios de las variables son estadísticamente significativas. Así, con un nivel de confianza del $95 \%$, el test $t$-Student para muestras independientes indica que el modelo de gestión adoptado por el servicio público autonómico de televisión influye en el share obtenido, la cifra total de activos, la subvención pública recibida, los gastos en personal, los gastos en materiales y el resultado económico.

\begin{tabular}{|l|r|r|r|r|}
\hline \multicolumn{1}{|c|}{ Variable de comparación } & \multicolumn{1}{c|}{ Modelo clásico } & \multicolumn{1}{c|}{$\begin{array}{c}\text { Modelo } \\
\text { externalizado }\end{array}$} & \multicolumn{1}{c|}{ T-value } & \multicolumn{1}{c|}{ p-value } \\
\hline (x1) Share & 9.62 & 6.19 & 45.99 & $0.000^{*}$ \\
\hline (x2) Activo total & $100,498,418.00$ & $22,958,879.40$ & -6.392 & $0.000^{*}$ \\
\hline (x3) Subvención pública recibida & $110,274,256.00$ & $13,811,597.00$ & -3.748 & $0.001^{*}$ \\
\hline (x4) Gastos en personal & $61,334,272.71$ & $45,577,149.85$ & -7.736 & $0.000^{*}$ \\
\hline (x5) Gastos en materiales & $45,447,029.54$ & $20,771,343.61$ & -3.448 & $0.000^{*}$ \\
\hline (x6) Resultado económico & $-57,518,753.40$ & $-24,456,577.10$ & 32.379 & $0.011^{*}$ \\
\hline * Significativo para $\mathrm{p}<0,05$ & & &
\end{tabular}

Tabla 3. Análisis de diferencias de medias según el modelo de gestión (2010-2013) 


\subsection{Análisis de productividad}

En la Tabla 4 se recogen los valores de la productividad obtenida por las cadenas autonómicas según el modelo de gestión utilizado. Estos valores indican la efectividad de una empresa para obtener productos y servicios (Brinkerhoff \& Dressler, 1990) desde la utilización de diferentes variables como el volumen de activos, el capital recibido, el factor trabajo utilizado o los materiales (Picard, 2003).

Como puede observarse, cada punto de share obtenido por los entes públicos con un modelo de gestión clásico ha requerido un volumen de activos 3 veces superior al que han necesitado los entes que utilizan un modelo de externalización de su actividad.

Además, para el grupo de televisiones autonómicas con un modelo de gestión clásico cada punto de share durante el periodo 2010-2013 ha supuesto al Gobierno autonómico correspondiente un desembolso 5 veces superior con respecto al realizado para el grupo de cadenas con un modelo de gestión basado en la externalización.

Con respecto a la relación entre los gastos en personal y el share obtenido, se observa que los entes públicos que apuesta por la externalización requieren para obtener un punto se share 1 millón de euros más en gastos de personal que los entes públicos clásicos. Sin embargo, los servicios públicos autonómicos de televisión que utilizan un modelo de gestión clásico han desembolsado por cada punto de share 1.5 millones de euros más en gastos de materiales que los servicios públicos autonómicos de televisión con un modelo de gestión basado en la externalización.

\begin{tabular}{|l|r|r|}
\cline { 2 - 3 } \multicolumn{1}{c|}{} & Modelo clásico & Modelo externalizado \\
\hline (x2) Activo total / Share (x3) & $10,446,821$ & $3,709,027.37$ \\
\hline (x3) Subvención pública recibida / (x1) Share & $11,463,020.37$ & $2,231,275.77$ \\
\hline (x4) Gastos en personal / (x1) Share & $6,375,704.02$ & $7,363,029.05$ \\
\hline (x5) Gastos en materiales / (x1) Share & $4,724,223.445$ & $3,355,629.02$ \\
\hline
\end{tabular}

Tabla 4. Productividad según el modelo de gestión (2010-2013)

\section{Conclusiones}

Los servicios públicos de televisión se están viendo actualmente afectados por numerosas amenazas de diversa naturaleza. Dentro de dichas amenazas, destaca la crisis económica que cuestiona la sostenibilidad de los entes públicos desde los ingresos y gastos derivados de su actividad, y el déficit económico generado. 
En este contexto se ha desarrollado este trabajo que analiza la influencia que tiene el modelo de gestión adoptado por el servicio público de televisión para gestionar su actividad productiva sobre diversas variables económicas.

Los resultados de la investigación señalan que los entes televisivos públicos que utilizan un modelo de gestión basado en externalizar parte de su actividad productiva presentan menores pérdidas económicas y requieren de una menor asignación pública de los Gobiernos correspondientes. Además, esta estrategia de gestión consigue unos niveles de productividad mucho más óptimos que los obtenidos por el modelo de gestión opuesto a la externalización. En este sentido, las corporaciones públicas que apuestan por la externalización necesitan menores niveles de activos y menores subvenciones económicas por cada punto de share obtenido.

Sin embargo, la externalización como estrategia de gestión también presenta ciertas desventajas que hay que señalar. Así, los servicios públicos de televisión con una estrategia de externalización obtienen en términos absolutos una cuota de pantalla mucho menor, y requieren de un mayor desembolso en gastos de personal para obtener un punto de share. Además, tal y como señala la literatura, la externalización puede tener importantes consecuencias negativas que hay que valorar cuando se considera este modelo de gestión.

Por último, este trabajo presenta ciertas limitaciones que deben ser consideradas. En este sentido, hay que indicar que la investigación realizada ha analizado un único país para un periodo temporal de 4 años y ha incluido solamente cadenas autonómicas. Por este motivo, las futuras líneas de trabajo deberían ir dirigidas a analizar la gestión económica de los servicios públicos de televisión de otros países europeos con periodos temporales más largos. Además puede ser muy relevante ampliar dicho análisis a cadenas de televisión públicas de ámbito local y nacional.

\section{Referencias}

Accenture (2012). Informe sobre el papel de la televisión pública autonómica en España. Madrid.

Alm, A., \& Ferrell Lowe, G. (2003). Outsourcing core competencies?. En G. Ferrel Lowe \& T. Hujanen (eds.), Broadcasting and convergence: New articulations of the public service remit. Goterborg: Nordicom.

Artero, J.P., Orive, V., \& Latorre, P. (2015). Efficiency and benchmarks of regional public service broadcasters in Spain. Communication \& Society, 28(3), 13-30. http://dx.doi.org/10.15581/003.28.3.13-28 
Bardoel, J., \& D’Haenens, L. (2008). Reinventing public service broadcasting in Europe: Prospect, promises and problems. Media, Culture and Society, 30(3), 337-355.

http://dx.doi.org/10.1177/0163443708088791

Brinkerhoff, R., \& Dressler, D. (1990). Productivity Measurament. A Guide for Managers and Evaluators. London: Sage.

Bustamante, E. (2009). El audiovisual digital: La televisión regional ante el reto de futuro. En E. Bustamante (Ed.), El audiovisual digital. Politicas y estrategias desde las Comunidades Autónomas. Tenerife: IDECO.

Campos, F. (2012). Modelo de financiación de las televisiones autonómicas. En J.C. Miguel \& M.A Casado (coords.), Televisiones autonómicas. Evolución y crisis del modelo público de proximidad. Gedisa: Barcelona.

Campos-Freire, F. (2013). El futuro de la TV europea es híbrido, convergente y cada vez menos público. Revista Latina de Comunicación Social, 68, 89-118. http://dx.doi.org/10.4185/RLCS-2013-970

Collins, R., Finn, R., Mcfadyen, S., \& Hoskins, C. (2001). Public service broadcasting beyond 2000: Is there a future for public service broadcasting?. Canadian Journal of Communication, 26(1), 3-15.

Cuesta, U. (2011). Las televisiones autonómicas en el nuevo panorama audiovisual. Documento para el debate. Madrid: Fundación Ciudadanía y Valores. Disponible online en: http://www.funciva.org/uploads/ficheros documentos/1304077495 ponencia ii.pdf

Fernández Alonso, I. (2002). La externalización de la producción de los operadores públicos de televisión de ámbito autonómico en España. Los casos canario y extremeño. Revista Latina de Comunicación Social, 46. Available online at::

http://www.ull.es/publicaciones/latina/2002/latina46enero/4604isabel.htm

Fernández Alonso, I., \& Fernández Viso, A. (2012). Internal pluralism in the governance of public service broadcasters in Spain and the role of social groups and professionals. The case of RTVE. Communication \& Society, 25(2), 203-230.

Kantar Media (2014). Informe anual de la audiencia de televisión. Madrid

Iosifidis, P. (2010). Review: M.Debrett, Reinventing public service televisión for digital future. International Journal of Digital Television, 2(1), 1-3. http://dx.doi.org/10.1386/jdtv.2.1.123_5

López-Vidales, N., Azurmendi, A., \& Órtiz, M.Á. (2012). Hacia un nuevo marco regulatorio y de gestión de la televisión pública en España: El bienio del cambio. Revista de Comunicación de la SEECI, 27, 39-59. http://dx.doi.org/10.15198/seeci.2012.27.39-59 
Mcivor, R., Humphreys, P.K, \& Mcaleer, W. (1997). A strategic model for the formulation of and effective make or buy decision. Management Decision, 35(2), 169-178.

http://dx.doi.org/10.1108/00251749710160331

Miguel De Bustos, J.C., \& Casado, M.A. (2012). Televisiones autonómicas: Evolución y crisis del modelo público de proximidad. Barcelona: Gedisa.

Muñoz, M. (2011). Key issues to understand the controversy surrounding the economic support of Spanish public television. Communication \& Society, 24(1), 119-140.

Picard, R. (2003). Assessment of Public Service Broadcasting: Economic and Managerial Performance Criteria. Javnost - The Public, 10(3), 29-44. http://dx.doi.org/10.1080/13183222.2003.11008833

Sánchez, J., \& Sarabia, I. (2012). Las estructuras de Gobierno de los nuevos modelos de televisión autonómica pública en España. Gestión y Análisis de las Políticas Públicas, 8, 35-61.

Intangible Capital, 2016 (www.intangiblecapital.org)

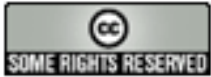

Article's contents are provided on an Attribution-Non Commercial 3.0 Creative commons license. Readers are allowed to copy, distribute and communicate article's contents, provided the author's and Intangible Capital's names are included. It must not be used for commercial purposes. To see the complete license contents, please visit http://creativecommons.org/licenses/by-nc/3.0/. 